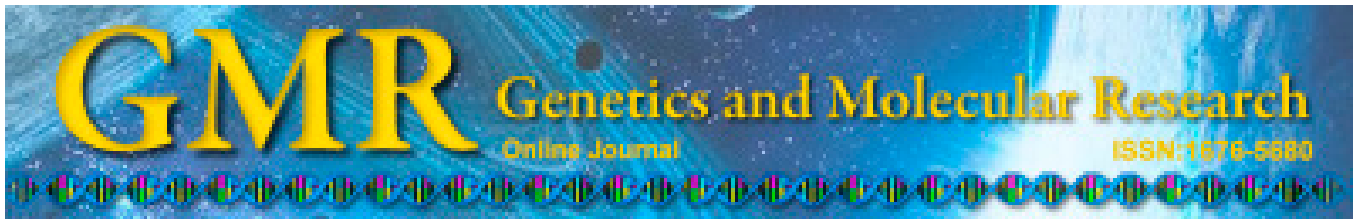

\title{
$P U M A$ gene transfection can enhance the sensitivity of epirubicin-induced apoptosis of MCF-7 breast cancer cells
}

\author{
C.-G. Sun ${ }^{1 *}$, J. Zhuang ${ }^{1 *}$, W.-J. Teng ${ }^{1}$, Z. Wang ${ }^{2}$ and S.-S. Du ${ }^{3}$ \\ ${ }^{1}$ Department of Oncology, Weifang Traditional Chinese Hospital, \\ Weifang City, Shandong, China \\ ${ }^{2} \mathrm{Xi}$ Yuan Hospital of the China Academy of Chinese Medical Sciences, \\ Beijing, China \\ ${ }^{3}$ Beijing Normal University, Beijing, China \\ *These authors contributed equally to this study. \\ Corresponding authors: Z. Wang / S.-S. Du \\ E-mail: wangzhong_w@163.com / dushushan_dss@yeah.net
}

Genet. Mol. Res. 14 (2): 5742-5749 (2015)

Received July 3, 2014

Accepted December 5, 2014

Published May 29, 2015

DOI http://dx.doi.org/10.4238/2015.May.29.6

\begin{abstract}
We explored whether p53 upregulated modulator of apoptosis (PUMA) gene transfection could enhance the sensitivity of epirubicin-induced apoptosis of MCF-7 breast cancer cells. The liposome-mediated recombinant eukaryotic expression vector PUMA-pCDNA3 and empty vector plasmid were stably transfected into MCF-7 cells. Epirubicin (0.01-100 $\mu \mathrm{M})$ was applied to MCF-7, MCF7/PUMA, and MCF-7/pCDNA3 cells for $72 \mathrm{~h}$. The MTT assay was used to calculate the cell survival rate in each group, and the $50 \%$ inhibitory concentration (IC50) was calculated. The IC50 values of epirubicin in MCF-7, MCF-7/PUMA, and MCF-7/pCDNA3 cells were $13 \pm 1.4,1.8 \pm 0.2$, and $10.7 \pm 1.3 \mu \mathrm{M}$, respectively. The sensitivity of MCF-7/PUMA cells to epirubicin increased 7.2-fold. Epirubicin induced apoptosis in MCF-7 cells dose-dependently, but MCF-7/PUMA cell-induced apoptosis was more significant compared to controls. Low
\end{abstract}


concentrations of epirubicin $(0.1 \mu \mathrm{M})$ caused low levels of apoptosis of MCF-7/pCDNA3 $(1.15 \pm 0.26 \%)$ and MCF-7 cells $(0.9 \pm 0.24 \%)$, but significantly induced apoptosis of MCF-7/PUMA cells (6.44 $\pm 1.46 \%)$. High epirubicin concentration $(1 \mu \mathrm{M})$ induced apoptosis in each group, but the epirubicin MCF-7/PUMA apoptosis rate $(35.47 \pm 9.36 \%)$ was significantly higher than that of MCF-7 (12.6 $\pm 3.73 \%)$ and MCF-7/ pCDNA3 $(15.2 \pm 5.17 \%)$ cells $(\mathrm{P}<001)$. Flow cytometry and TUNEL assays for apoptosis detection showed similar results. PUMA protein expression in MCF-7/PUMA cells was significantly higher than that in MCF-7 and MCF-7/pCDNA3 cells by Western blot analysis. Therefore, stable transfection of PUMA can significantly enhance epirubicininduced apoptosis sensitivity of MCF-7 breast cancer cells.

Key words: PUMA gene; Breast cancer; MCF-7 cells; Apoptosis

\section{INTRODUCTION}

Breast cancer is the most common malignancy in women and its incidence has shown a clear upward trend in recent years ( $\mathrm{Li}$ et al., 2012). Breast cancer is now widely recognized as a complex disease that has both gene and environmental interactions. The traditional method of treating breast cancer consists of surgical resection, radiotherapy, chemotherapy, and endocrine therapy. With a deeper understanding of the pathogenesis of human malignancies and the rapid development of molecular biology techniques, gene therapy has become an important direction to explore regarding the biological treatment of breast cancer (Ma et al., 2009). A newly discovered and reported pro-apoptotic member in the Bcl-2 protein family in recent years is the p53 upregulated modulator of apoptosis (PUMA), which has a strong pro-apoptotic effect. Studies have shown that PUMA plays an important role in the apoptosis of cells and in tumorigenesis in p53-dependent and independent pathways. PUMA is both more powerful and more stable than p53 in its pro-apoptotic effects; therefore, the study of gene therapy has received widespread attention in recent years (Wang et al., 2009). In vitro experiments have shown that exogenous PUMA gene transfection can induce the rapid apoptosis of tumor cells and inhibit colony formation. PUMA gene transfection in vivo significantly increased chemotherapy and radiation sensitivity of esophageal cancer, head and neck carcinoma, choriocarcinoma, and enhanced the therapeutic effect of these treatments (Chen et al., 2007; Sun et al., 2007).

\section{MATERIAL AND METHODS}

\section{Cell lines and reagents}

PUMA-pCDNA3 and pCDNA3 were purchased from Shanghai Biological Technology Co., Ltd. (Shanghai, China). Mouse anti-human PUMA polyclonal antibody was purchased from Cell Signaling Technology (Danvers, MA, USA). G418 and Lipofectamine 2000 were purchased from Gibco (Gaithersburg, MD, USA). Western blotting, terminal deoxynucleotidyl transferase-dUTP nick-end labeling (TUNEL), 3-(4,5-dimethylthiazol-2-yl)-2,5-diphenyltetrazolium bromide (MTT), and fast extraction kits for small quantity plasmid purification were purchased from Shanghai Biological, 
Hua Shungong Division. Protein molecular weight standards were purchased from the Shanghai Institute of Biochemistry (Shanghai, China). Breast cancer cell line MCF-7 cells were purchased from the Shanghai Cell Department of the Chinese Sciences Academy (Shanghai, China). A 1640 culture medium containing 10\% fetal calf serum was used. The culture conditions were at $37^{\circ} \mathrm{C}$ and saturated humidity and $5 \% \mathrm{CO}_{2}$. Cells in logarithmic growth were chosen for experimental use.

\section{Cell transfection}

MCF-7 cells in logarithmic growth phase were resuspended in $2 \mathrm{~mL}$ complete culture medium were seeded on 6-well plates with a density of $2 \times 10^{5} /$ well at $37^{\circ} \mathrm{C}$ in a $5 \% \mathrm{CO}_{2}$ incubator overnight. Cells were grown to approximately $60-80 \%$ confluence. The culture medium without serum and antibiotics was used for washing, and $2 \mu \mathrm{L}$ purpose plasmid PUMA-pCDNA3 or empty vector plasmid pCDNA3 and $8 \mu \mathrm{L}$ liposomes were added on each well and placed in a $\mathrm{CO}_{2}$ incubator at $37^{\circ} \mathrm{C}$ for $5 \mathrm{~h}$. The culture medium was replaced with Dulbecco's modified Eagle medium containing $20 \%$ fetal bovine serum. After culture for $72 \mathrm{~h}$ in the culture medium, G418 medium ( $3 \mathrm{mg} / \mathrm{mL} \mathrm{G418)} \mathrm{was} \mathrm{used} \mathrm{for}$ culture and screening. The liquid was changed every 3-5 days. Two weeks later, a plurality of cloned single cells was selected from the cells with stable expression for further culture. The PUMA-pCDNA3- and pCDNA3-transfected cells were labeled MCF-7/PUMA and $\mathrm{MCF}-7 / \mathrm{pCDNA} 3$, respectively.

\section{MTT assay detection of PUMA/MCF-7 sensitivity towards epirubicin cytotoxicity}

The MCF-7, MCF-7/PUMA, and MCF-7/pCDNA3 cells were seeded on 96-well culture plates $\left(1 \times 10^{4} /\right.$ well). The next day, $100 \mu \mathrm{L}$ culture medium containing a series of concentrations of epirubicin $(0.01,0.1,0.5,1,5,10,20,50$, and $100 \mu \mathrm{M})$ was substituted on each well. After $48 \mathrm{~h}, 10 \mu \mathrm{L}$ MTT solution $(2.5 \mathrm{mg} / \mathrm{mL})$ was added on each well for 4 $\mathrm{h}$ of incubation. The culture medium was then removed, and $100 \mu \mathrm{L}$ dimethyl sulfoxide (Sigma-Aldrich, St. Louis, MO, USA) was added to each well. The crystals formed were dissolved and shaken for $5 \mathrm{~min}$. The optical density (OD) value was measured at $570 \mathrm{~nm}$ wavelength. The blank was set parallel with the experimental wells, and adjusted to zero following addition of media. The experiment was conducted three times. The results were averaged, with the cell survival rate $(\%)=($ experimental group OD - blank group OD)/ control group OD - blank group OD) x 100\%.

\section{Flow cytometric (FCM) detection of tumor cell apoptosis}

The MCF-7, MCF-7/PUMA, and MCF-7/pCDNA3 cells were seeded on 24-well culture plates $\left(1 \times 10^{4} /\right.$ well $)$ overnight. The next day, $1 \mu \mathrm{M}$ epirubicin was added. On the second day, $100 \mu \mathrm{L}$ cells were routinely cultured for $72 \mathrm{~h}$. Fluorescein isothiocyanate (FITC) and propidium iodide (PI) were added for double staining according to manufacturer instructions, and samples were placed for FCM. The control group was without epirubicin. Determination of results was as follows: the apoptotic cells can exclude dyes used for cell activity identification such as PI, but the necrotic cells do not have such ability. Therefore, DNA in cells with cell membrane damage could be stained with PI to show red fluorescence, whereas cells with 
intact membranes would not fluoresce. Thus, in early apoptotic cells and in viable cells, PI would not yield red fluorescence signals. In a bivariate FCM scatter plot, the live cells are displayed in the lower left quadrant (FITC-PI-). Inactive cells, which are necrotic cells, are displayed in the upper right quadrant (FITC/PI). Apoptotic cells are displayed in the lower right quadrant (FITC/PI-).

\section{TUNEL assay detection of epirubicin-induced apoptosis of tumor cells}

The cells in each group were centrifugally resuspended and inoculated to a $3.5-\mathrm{cm}$ culture dish with an inner cover glass $\left(1 \times 10^{8} /\right.$ dish $)$. Epirubicin $(0.1$ or $1 \mu \mathrm{M})$ was added after incubation at $37^{\circ} \mathrm{C}$ for $24 \mathrm{~h}$. The cells $(10 \mu \mathrm{L})$ were incubated for $72 \mathrm{~h}$. The control group was without epirubicin. The TUNEL kit was used. According to kit instructions, the number of apoptotic cells was calculated per high-power field. Five fields were counted for each sample.

\section{Western blot detection of PUMA expression in tumor cells}

At $72 \mathrm{~h}$, the total cellular protein, with or without epirubicin, was used for Western blotting. The sodium dodecyl sulfate-polyacrylamide gel electrophoresis sample volume was $15 \mu \mathrm{g} / \mathrm{L}$ under the following conditions: constant, 20-40 mA in each plate, with electrophoresis time approximately $2 \mathrm{~h}$. The transfer to film conditions were as follows: constant, $42 \mathrm{~mA}$ in each film, $3.5 \mathrm{~h}$.

\section{Statistical analysis}

All data results were analyzed with the SPSS 17.0 statistical software (Chicago, IL, USA). Rates were compared using the $\chi^{2}$ test. The continuous data are reported as means \pm SD. Means between two independent samples were compared using the Student $t$-test. Analysis of variance was used for multiple group comparison. Significance was considered at $\mathrm{P}<0.05$.

\section{RESULTS}

Transfer of the PUMA gene into breast cancer cells may enhance the sensitivity of the apoptosis effects of epirubicin

The MTT assay was used to detect the sensitivity of apoptosis effects of epirubicin on breast cancer cells. It was found that when the epirubicin concentration ranged from 0.01 to $100 \mu \mathrm{M}$, there was dose dependence between epirubicin concentrations and cell viability. With the increase of epirubicin concentration, cell viability decreased. Compared with that of MCF-7 and MCF-7/pCDNA3 cells, the cell viability of MCF-7/PUMA decreased more significantly. The Excel software was used to calculate the IC50 values of MCF-7 and MCF$7 /$ pCDNA3 cells, which were $13 \pm 1.4$ and $10.7 \pm 1.3 \mu \mathrm{M}$, respectively. In contrast, MCF-7/ PUMA, with an IC50 of $1.8 \pm 0.2 \mu \mathrm{M}$, was 7.2 times more sensitive than MCF-7 cells (Figure 1). Thus, $P U M A$ gene transfection in breast cancer cells could increase the sensitivity of epirubicin-induced apoptosis. 

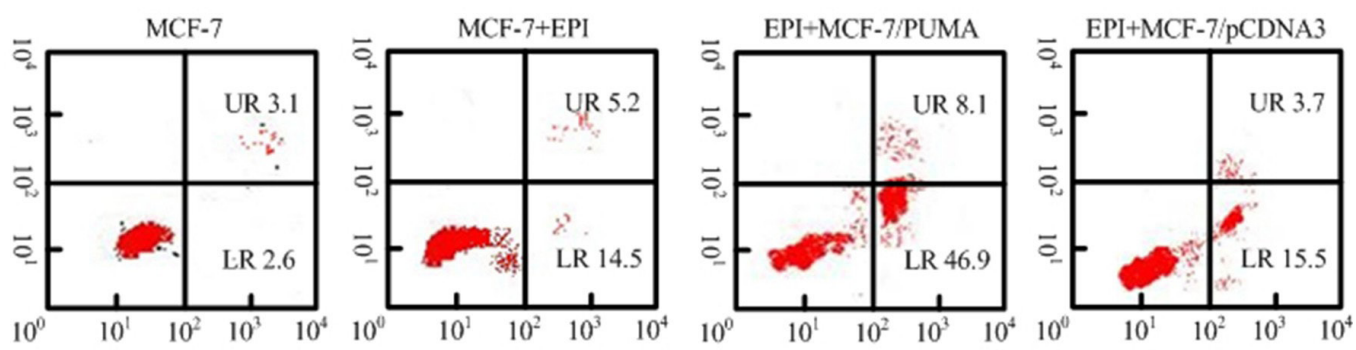

Figure 1. Impact of PUMA gene transfection in enhancing the sensitivity of MCF-7 cells to pirarubicin cytotoxicity. UR: upper right; LR: lower right.

\section{FCM test assessment of MCF-7/PUMA apoptosis}

The cells were marked with Annexin V-PI and placed on FCM for apoptosis detection. Figure 2 shows that the MCF-7 cell apoptosis rate was $5.50 \pm 1.03 \%$. After the use of epirubicin $(1 \mu \mathrm{M})$, the MCF-7 cell apoptosis rate was $18.1 \pm 2.13 \%$. On the other hand, after the use of epirubicin $(1 \mu \mathrm{M})$, the MCF-7/PUMA cell apoptosis rate was $52.18 \pm 10.2 \%$, which was significantly higher than that of the MCF-7 group $(\mathrm{P}<0.01)$ and the MCF-7/PUMA group $(\mathrm{P}<0.05)$. After the use of epirubicin, the MCF-7/pCDNA3 cell apoptosis rate was $18.55 \pm 4.32 \%$, which was not significantly different from that of MCF-7/epirubicin cells at $18.1 \pm 2.13 \%(\mathrm{P}>0.05)$. This suggests that $P U M A$ transfection can enhance the pro-apoptotic effects of epirubicin.

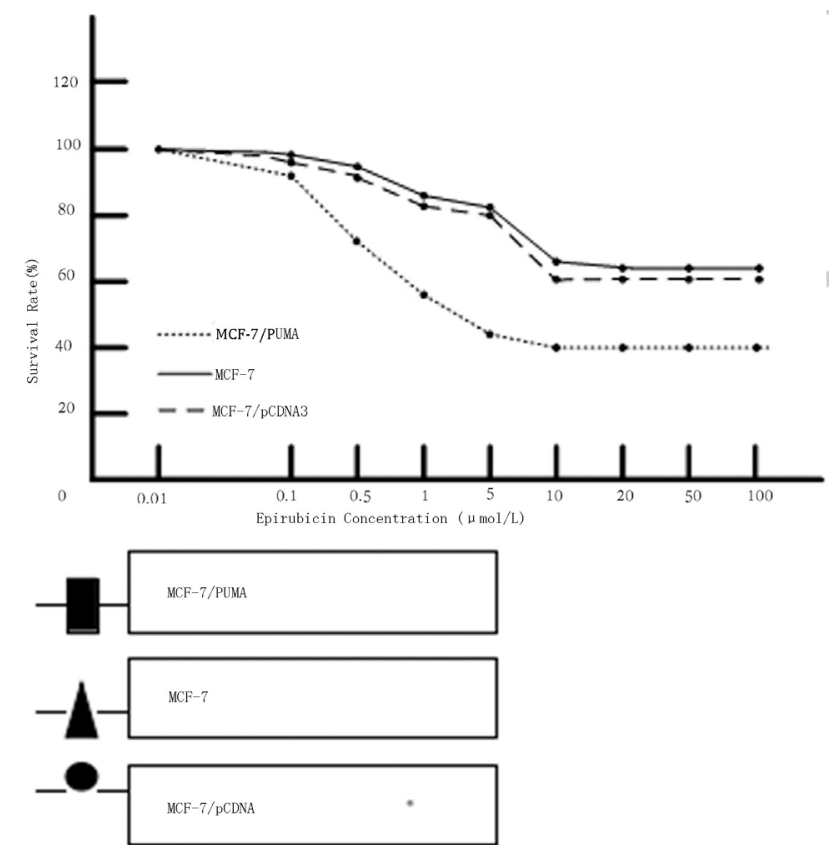

Figure 2. Flow cytometric analysis of pirarubicin-induced apoptosis in MCF-7/PUMA cells. Live cells are displayed in the lower left quadrant (FITC-PI-). Inactive cells, which are necrotic cells, are displayed in the upper right quadrant (FITC/PI). Apoptotic cells are displayed in the lower right quadrant (FITC/PI-). 


\section{TUNEL assay assessment of MCF-7/PUMA apoptosis}

After the use of $0.1 \mu \mathrm{M}$ epirubicin for $72 \mathrm{~h}$, the MCF-7/PUMA apoptosis rate was $6.44 \pm 1.46 \%$, whereas the apoptotic rates of MCF-7 and MCF-7/pCDNA3 cells were $0.9 \pm$ $0.24 \%$ and $1.15 \pm 0.26 \%$, respectively. After $72 \mathrm{~h}$ of $1 \mu \mathrm{M}$ epirubicin use, apoptotic cells were observed in the cells of each group. The apoptosis rates of MCF-7 and MCF-7/pCDNA3 were $12.6 \pm 3.73 \%$ and $15.2 \pm 5.17 \%$, respectively, whereas the apoptosis rate of MCF-7/PUMA was $35.47 \pm 9.36 \%$. The differences were statistically significant $(\mathrm{P}<0.05)$.

\section{$P U M A$ transfection and epirubicin-induced PUMA protein expression changes in breast cancer cells}

To determine whether PUMA transfection is associated with epirubicin-induced apoptosis, we analyzed PUMA protein changes. MCF-7 cells contain a small amount of endogenous PUMA protein expression. PUMA protein expression increased after the use of epirubicin. After epirubicin was used in MCF-7/PUMA cells, PUMA expression increased more significantly. After epirubicin was used in MCF-7/pCDNA3 cells, PUMA protein expression was similar with that in the MCF-7/epirubicin group (Figure 3).

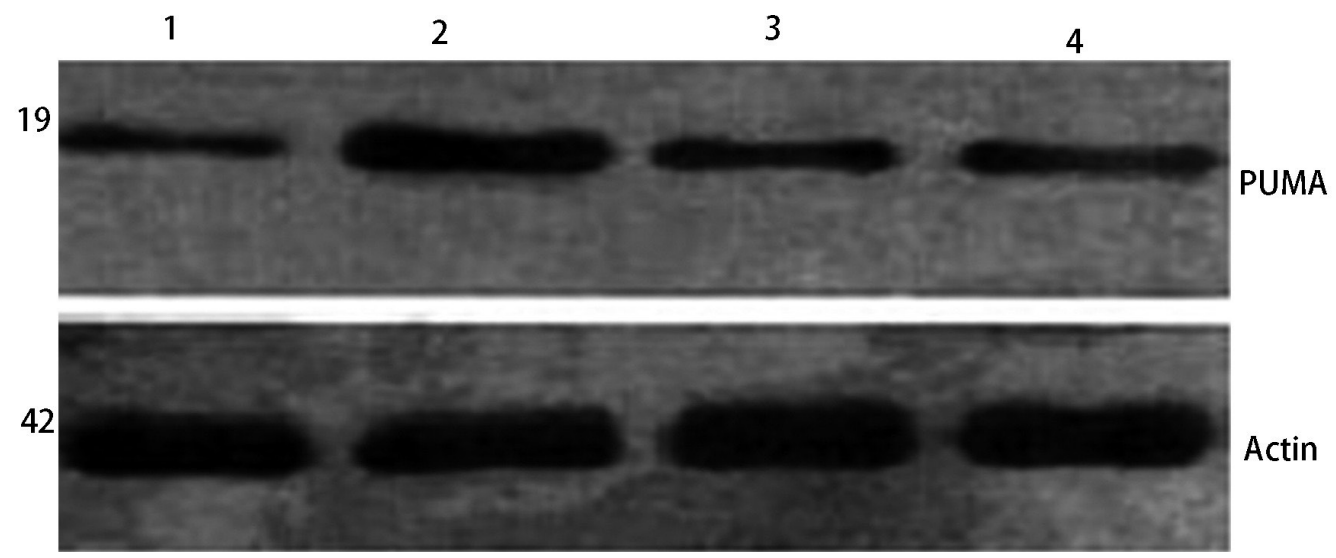

Figure 3. Pirarubicin-induced PUMA protein expression in MCF-7/PUMA cells. 1) MCF-7; 2) MCF-7/PUMA+ epirubicin (EPI) 3) MCF-7/pCDNA3+ epirubicin (EPI); 4) MCF-7+ epirubicin (EPI). Protein size (kDa) is indicated to the left of the gel image.

\section{DISCUSSION}

Breast cancer ranks first in incidence among women. As living standards have improved, the incidence of breast cancer has also increased and has shown a younger age trend. Breast cancer has become the number one killer in women's health. At present, the main treatment is surgery combined with anthracycline chemotherapy. Epirubicin is an anthracycline compound. It is a broad-spectrum anti-tumor clinical antibiotic, its main function to achieve the killing effect of tumor cells being to embed directly between DNA base pairs to interfere with transcription and prevent the formation of mRNA (Sun and Zhou, 1996). However, in the majority of tumor chemotherapy cases, many chemotherapy regimens containing anthracy- 
cline reach drug resistance or a cumulative cardiac toxicity dose. Breast cancer is the result of tumor cell proliferation and apoptosis imbalances. The loss of cell cycle control leads to cell proliferation and apoptosis blockage, and results directly in aging, damage, and the prolonged life of abnormal cells. Therefore, finding drugs that increase chemosensitivity has become a research hotspot. There are many methods to enhance the sensitivity of anthracycline-epirubicin chemotherapy. In addition to the combination of traditional chemotherapy drugs, epirubicincombined gene therapy is a new method to enhance the sensitivity of epirubicin and has achieved remarkable results. PUMA is the apoptosis-promoting gene downstream of $p 53$. Decrease or loss of its expression has been observed in many tumor cells (Li et al., 2009; Lui et al., 2010; Zhou and Zhou, 2010; Song et al., 2012). Increasing intracellular PUMA expression can promote tumor cell apoptosis and can significantly enhance the sensitivity of tumor cells to chemotherapy, suggesting that PUMA might be a chemotherapy sensitizer for tumor therapy.

This study explored whether PUMA gene transfection can enhance the sensitivity of MCF-7 breast cancer cells to epirubicin treatment. It was found that when a series of concentrations of epirubicin was used in MCF-7/PUMA cells, PUMA could significantly enhance the sensitivity of MCF-7 cells to epirubicin treatment and might lower the IC50 average. Whether epirubicin induced apoptosis was further analyzed. The results showed that $0.1 \mu \mathrm{M}$ epirubicin only induced PUMA-transfected cells to apoptose; it could not induce apoptosis of control MCF-7 cells or those receiving vector alone. High concentrations of epirubicin (1 $\mu \mathrm{M})$ could induce apoptosis in all cell lines. However, the apoptosis rate in $P U M A$-transfected cells was significantly higher than that in non-PUMA-transfected MCF-7 cells and empty vector-transfected MCF-7 cells. The results suggest that PUMA might enhance epirubicininduced apoptosis in MCF-7 cells. We also analyzed PUMA protein expression. MCF-7 cells were found to contain only a small amount of endogenous PUMA expression. After the use of epirubicin, PUMA protein expression increased. After the PUMA gene-transfected cells were under the effects of epirubicin, PUMA protein expression increased more significantly. Hence, epirubicin chemotherapy sensitivity was enhanced by increasing the expression of PUMA. Therefore, the development of DNA elements, transcription factors, and other non-toxic small molecules could lead to corresponding PUMA activation. These substances could activate the strong expression of PUMA in tumor cells, and provide a potential mechanism to enhance epirubicin chemotherapy sensitivity. Therefore, our results suggest a new direction for development of breast cancer treatments.

\section{REFERENCES}

Chen Y, Qian H, Wang H, Zhang X, et al. (2007). Ad-PUMA sensitizes drug-resistant choriocarcinoma cells to chemotherapeutic agents. Gynecol. Oncol. 107: 505-512.

Li K, Li SZ, Zhang YL, Zhang YH, et al. (2009). Effect of Quercetin on proliferation and invasion of human breast carcinoma cell line MDA - MB - 435s. Cancer Res. Prev. Treat. 36: 549-551.

Li YY, Zhang B, Zhao HM, Feng WH, et al. (2012). The effects of PXD101 on proliferation and apoptosis of human breast cell line MCF-7 and its mechanism. Chin. J. Clin. Oncol. 39: 249-253.

Lui F, Sun G, He C, He XG, et al. (2010). Effect of siRNA mediated silencing of cFLIP gene combined with epirubicin on apoptosis of MCF-7 cells. J. Pract. Oncol. 24: 337-340.

Ma BL, Geng ZL, Alibivati AN, Xu X, et al. (2009). 15dPGJ2 combined pcDNA3.0-PTEN plasmid transfection interefered MCF-7 cell line in vitro. J. Xinjiang Med. Univ. 32: 833-838.

Song JY, Zhang LN, Zheng H and Chen KX (2012). Expression of apoptosis suppressor gene AVEN in breast cancer patients. Chin. J. Clin. Oncol. 39: 197-200.

Sun Y and Zhou JC (1996). Editor in chief, Handbook of clinical oncology. 3rd edn. Beijing, People's Health Publishing House, pp 348-353. 
Sun Q, Sakaida T, Yue W, Gollin SM, et al. (2007). Chemosensitization of head and neck cancer cells by PUMA. Mol. Cancer Ther. 6: 3180-3188.

Wang R, Wang X, Li B, Lin F, et al. (2009). Tumor-specific adenovirus-mediated PUMA gene transfer using the survivin promoter enhances radiosensitivity of breast cancer cells in vitro and in vivo. Breast Cancer Res. Treat. 117: 45-54.

Zhou YH and Zhou SF (2010). Cox-2 expression of breast cancer with paclitaxel liposome combined table soft than star the findings of a study of neoadjuvant chemotherapy curative effect. J. Clin. Oncol. 15: 435-437. 